\title{
Evaluación de la eficacia clínica de tres protocolos antibióticos en yeguas diagnosticadas con endometritis bacteriana
}

Evaluation of the clinical efficacy of three antibiotic protocols in mares diagnosed with bacterial endometritis

Gallego-Rodríguez RS, Ruíz-Jaramillo AF, Ruíz Buitrago JD. Evaluación de la eficacia clínica de tres protocolos antibióticos en yeguas diagnosticadas con endometritis bacteriana. Rev Colombiana Cienc Anim. Recia. 2021; 13(1):e772. https://doi.org/10.24188/recia.v13.n1.2021.772

Universidad de Sucre, Colombia

Los autores permiten a RECIA reimprimir el material publicado en él. En caso de que un autor quiera traducir o usar una publicación parcial o completa de nuestro Diario, el autor debe obtener un permiso por escrito del editor de la revista.

Copyright (c) 2021. El (los) autor (es). Este es un artículo de acceso abierto distribuido bajo los términos de Creative Commons Attribution 4.0 (https://creativecommons.org/licenses/by-nc$\underline{\mathrm{sa} / 4.0 /)}$, El uso, distribución o reproducción está permitido, siempre que se acrediten al autor original y al propietario del copyright y que se cite la publicación original en esta revista, de acuerdo con la práctica académica aceptada. No se permite el uso, distribución o reproducción que no cumpla con estos términos. 


\title{
Evaluación de la eficacia clínica de tres protocolos antibióticos en yeguas diagnosticadas con endometritis bacteriana
}

\author{
Evaluation of the clinical efficacy of three antibiotic protocols in mares diagnosed with \\ bacterial endometritis
}

Renso Sneider Gallego Rodríguez. M.Sc.

Práctica Privada y académica en Medicina Interna y Reproducción en

DOI: https://doi.org/10.24188/recia.v13.n1.2021.772

Equinos.

renso1287@gmail.com

(D) https://orcid.org/0000-0003-1563-9731

Andrés Felipe Ruíz Jaramillo. M.Sc.

Práctica Privada y académica en Medicina Interna y Reproducción en

Equinos.

rujandres@gmail.com

(D) https://orcid.org/0000-0002-7985-7773

Jhon Didier Ruíz Buitrago. Ph.D.

Universidad CES, Facultad de Medicina Veterinaria y Zootecnia, Grupo

de Investigación en Veterinaria (INCA-CES), Medellín, Colombia.

jdruiz@ces.edu.co

Dhttps://orcid.org/0000-0003-4410-4349

Recepción: Julio 2020

Aprobación: Marzo 2021

Publicación: Abril 2021

\section{RESUMEN}

La endometritis bacteriana es una de las causas más comunes de falla reproductiva en yeguas, generalmente asociada a agentes bacterianos como Streptococcus equi, Echerichia coli, Enterococcus spp, Klebsiella sp., entre otros. De manera común se utilizan terapias antimicrobianas a base de fármacos betalactámicos y aminoglucósidos infiltrados a nivel uterino o en terapia sistémica. Para el presente estudio se tiene como objetivo principal evaluar la eficacia clínica de tres protocolos antibióticos en yeguas con diagnóstico de endometritis bacteriana y verificar su desempeño reproductivo postratamiento. La muestra se realizó con 90 yeguas diagnosticadas con infección uterina. Mediante examen clínico reproductivo, vaginoscopia y ecografía. Se tomaron muestras de cultivo uterino por medio de hisopado, a su vez se realizó antibiograma. Las yeguas fueron divididas en tres grupos de tratamiento antibiótico asignados según el resultado del antibiograma: penicilina G procaínica $(n=4)$, ceftiofur $(n=10)$ y gentamicina $(n=20)$. Posteriormente se evaluó la eficacia clínica y el desempeño reproductivo de las yeguas. De las tratadas con penicilina, 75\% (3/4) se recuperaron y dos quedaron gestantes; de las tratadas con gentamicina, 75\% (15/20) se recuperaron y 8 quedaron gestantes; en tanto que de las tratadas con ceftiofur, $70 \%(7 / 10)$ se recuperaron y a 5 se le confirmó gestación. Se concluye no hubo diferencias clínicas o reproductivas postratamiento por efecto del tipo de antibiótico empleado.

Palabras clave: Antibióticos; diagnóstico; endometritis; fertilidad; reproducción; yegua.

\begin{abstract}
Bacterial endometritis is one of the most common causes of reproductive failure in mares, generally associated with bacterial agents such as Streptococcus equi, Echerichia coli, Enterococcus spp, Klebsiella sp., Among others. Antimicrobial therapies based on beta-lactam drugs and aminoglycosides infiltrated at the uterine level or in systemic therapy are commonly used. The main objective of this study is to evaluate the clinical efficacy of three antibiotic protocols in mares with a diagnosis of bacterial endometritis and to verify their post-treatment reproductive performance. The sample was made with 90 mares diagnosed with uterine infection. By reproductive clinical examination, vaginoscopy and ultrasound.
\end{abstract}

Gallego-Rodríguez RS, Ruíz-Jaramillo AF, Ruíz Buitrago JD. Evaluación de la eficacia clínica de tres protocolos antibióticos en yeguas diagnosticadas con endometritis bacteriana. Rev Colombiana Cienc Anim. Recia. 2021; 13(1):e729. https://doi.org/10.24188/recia.v13.n1.2021.729 
Uterine culture samples were taken by means of swabbing, in turn an antibiogram was performed. The mares were divided into three antibiotic treatment groups assigned according to the antibiogram results: procaine penicillin $\mathrm{G}(\mathrm{n}=4)$, ceftiofur $(n=10)$ and gentamicin $(n=20)$. Subsequently, the clinical efficacy and reproductive performance of the mares were evaluated. Of those treated with penicillin, 75\% (3/4) recovered and two became pregnant; of those treated with gentamicin, 75\% (15/20) recovered and 8 became pregnant; while of those treated with ceftiofur, 70\% (7/10) recovered and 5 had pregnancy confirmed. It is concluded there were no post-treatment clinical or reproductive differences due to the type of antibiotic used.

Keywords: Antibiotics; diagnostics; endometritis; fertility; mare; reproduction.

\section{INTRODUCCIÓN}

La endometritis bacteriana es considerada una de las causas más comunes de infertilidad en la yegua, llegando a niveles de prevalencia entre 25 y $60 \%$ de yeguas infértiles (1). Generalmente los signos clínicos aparecen en yeguas que no conciben luego del servicio, que presentan reabsorción embrionaria, pérdidas tempranas fetales, aborto, placentitis o metritis posparto (2).

La infección endometrial se encuentra comúnmente relacionada con bacterias aeróbicas, tales como E. coli, Proteus spp, Staphylococcus aureus, Streptococcus spp, Pseudomonas aeruginosa y Klebsiella pneumoniae (3). Streptococcus equi zooepidemicus es la bacteria con mayor prevalencia en el útero de las yeguas (1). El diagnóstico clínico de la endometritis se realiza mediante el examen clínico y la palpación transrectal, así como mediante vaginoscopía, citología por medio del citocepillo, lavado de bajo volumen, cultivo del contenido del útero y biopsia del endometrio (4).

El empleo de antibióticos intrauterinos ha sido el método de elección para el manejo de problemas de infertilidad por causas infecciosas en las yeguas. En la práctica se recomienda la terapia intrauterina en lugar del tratamiento sistémico (2). Entre los antibióticos empleados se encuentra el ceftiofur, una cefalosporina de tercera generación de amplio espectro para varios de los agentes patógenos relacionados con esta patología, como es el caso de infecciones por Streptococcus zooepidemicus y E. coli (5). Así mismo, la gentamicina (aminoglucósido), habiéndose encontrado buena respuesta en concentraciones endometriales de 65.4-73.3 $\mu \mathrm{g} / \mathrm{ml} 24$ horas después de la infusión, no obstante, se debe tener precaución con las infusiones constantes o prolongadas, ya que pueden generar una fibrosis periglandular, comprometiendo la concepción (6).

Las vías de administración, las dosis y el agente bacteriano entre otros factores influyen en la elección del protocolo antibiótico para el tratamiento de la endometritis en yeguas (7). Por lo tanto, el objetivo del presente estudio fue evaluar la eficacia clínica de tres protocolos antibióticos en yeguas con diagnóstico de endometritis bacteriana y verificar su desempeño reproductivo postratamiento.

\section{MATERIALES Y MÉTODOS}

Consideraciones Éticas. El presente estudio fue aprobado en el acta 04/13 del Comité Institucional para el Cuidado y el Uso de Animales (CICUA) de la Universidad CES Medellín, Colombia.

Animales y Evaluación Clínica. El examen clínico de los animales fue realizado por un médico veterinario con especialidad y experiencia en el área reproductiva, cada evaluación fue hecha en el sitio donde se encontraban presentes los animales. Se consideraron 90 yeguas criollas colombianas, entre 4 y 12 años, condición corporal 2.5-4 (Según la escala propuesta por Webb y Weaver en 1979 la cual propone un puntaje de 1 siendo (muy pobre) a 6 siendo (muy gordo), por lo tanto puntajes entre 3 o 4 se consideran buenos), que hayan presentado repetición de celo e historial de subfertilidad, que no hayan sido tratadas con antibióticos por un mínimo de 30 días previos (revisión del médico veterinario tratante y/o historial clínico), y que tengan signos relacionados con endometritis bacteriana.

Clasificación de la endometritis. Para fines del presente estudio la endometritis fue clasificada se definió como el proceso de infección uterina con signos clínicos evidentes de descarga vaginal y acumulación de exudado en la comisura ventral de la vulva. Para la evaluación de la endometritis subclínica se tomó como el proceso de infección uterina sin signos clínicos evidentes, pero con historia clínica de infertilidad y con posterior diagnóstico a través de las pruebas utilizadas (examen clínico reproductivo, vaginoscopia, ecografía reproductiva, cultivo por hisopado uterino). 
Manejo de Muestras. La toma de la muestra se realizó con un hisopo estéril especial para la especie equina con una funda para mantener la esterilidad durante su paso por la vagina y el cérvix. Las muestras se obtuvieron de la porción dorsal y ventral de la pared del cuerpo del útero. La muestra se almacenó en un medio de transporte Stuart en refrigeración se envió al laboratorio para el análisis microbiológico según lo descrito por Anon 2009, en el Centro de Medicina Veterinaria y Zootecnia de la Universidad CES, en Medellín, en un tiempo de entrega que varió entre 30 minutos a dos horas.

Cultivo y Antibiograma. Las muestras se sembraron en agar MacConkey a $37^{\circ} \mathrm{C}$ durante 72 h. Para la clasificación de las colonias se utilizaron rutas bioquímicas (Agar-hierro-triple azúcar [TSI], lisina, urea, citrato y medio de Sulfuro Indol para Movilidad [SIM]). Luego se realizó el antibiograma para cada cultivo obtenido, utilizando discos de ceftiofur, penicilina G procaínica, gentamicina, amikacina, cefquinoma y ampicilina.

Tratamiento Antibiótico. El tratamiento se eligió con base en los resultados del antibiograma. En los casos de aislamiento de bacterias gram positivas (G+) se utilizó el protocolo de tratamiento intrauterino con penicilina G procaínica (Penicilina G Procaínica 5 millones de UI) ( $\mathrm{n}=4$ yeguas), en los casos de aislamiento de bacterias gram negativas (G-) se utilizó gentamicina (Gentavet ${ }^{\circledR} 5 \%, 1 \mathrm{~g}$ ) (n=20 yeguas), y en los casos de aislamientos de bacterias G+ y G- se utilizó ceftiofur (Cefur ${ }^{\circledR} 5 \%, 1.5 \mathrm{~g}$ ), antibiótico de amplio espectro ( $n=10$ yeguas), lo cual hace un total de 70 yeguas. El número de yeguas faltantes fueron tratadas con los otros antibióticos presentes en el antibiograma debido a que las bacterias aisladas presentaban resistencia a penicilina G, gentamicina y ceftiofur. Los antibióticos fueron diluidos en 100 ml de solución salina fisiológica estéril y fueron aplicados diariamente durante tres días. Todos los tratamientos se hicieron bajo el mismo protocolo, cada uno fue realizado por un período de cinco días con infusiones intrauterinas cada 24 horas.

Eficacia Clínica y Desempeño Reproductivo. Ocho días después de finalizado el tratamiento se volvió a realizar el examen del tracto reproductivo para determinar la posible presencia de secreción vulvar, flujo vaginal, así como edema uterino por medio de ecografía. Las yeguas que presentaron descarga vaginal mucopurulenta y edema uterino anormal se diagnosticaron con endometritis persistente. Solo fueron servidas (mediante inseminación artificial x 2 servicios) las yeguas que no presentaron ningún signo de infección uterina. Se confirmó la gestación 15 días después del servicio mediante ecografía transrectal.

Análisis Estadístico. Se realizaron medidas de estadística descriptiva en la cual se determinó la eficacia clínica y reproductiva de tres protocolos antibióticos en las yeguas que fueron diagnósticas con endometritis bacteriana por medio de una base de datos categorizada en Excel.

\section{RESULTADOS}

De las 90 muestras analizadas mediante cultivo bacteriano se encontró que 38 (42.22\%) presentaron asilamientos de bacterias gram negativas, a su vez 15 (16.67\%) tuvieron crecimiento de bacterias gram positivas, del total de yeguas 23 (25.56\%) presentaron infección mixta y en 14 (15.55\%) no hubo crecimiento bacteriano.

Del total de cultivos con bacterias gram negativas se encontró que estas bacterias fueron sensibles en 52.63\% (20/38) muestras para gentamicina, así mismo, de los cultivos con bacterias gram positivas se obtuvo que hubo sensibilidad a la penicilina G en el 26.66\% (4/15) de los casos, a su vez, de las muestras en las que se aislaron cultivos con infección mixta se encontró que hubo sensibilidad al ceftiofur en 43.47\% (10/23) de las muestras (Tabla 1).

Tabla 1. Evaluación de los valores relacionados a la eficacia clínica y la eficacia reproductiva en los tres protocolos antibióticos.

\begin{tabular}{ccccccccccc}
\hline Yegua & \multicolumn{4}{c}{ Eficacia Clínica } & \multicolumn{5}{c}{ Eficacia Reproductiva } \\
\hline Antibiótico & $\mathbf{n}$ & Sin Infección & \% & Infección Persistente & \% & Servidas & Preñez & \% & Vacía & \% \\
\hline Gentamicina & 20 & 15 & 75 & 5 & 25 & 13 & 8 & 61,54 & 5 & 38,46 \\
Penicilina G & 4 & 3 & 75 & 1 & 25 & 3 & 2 & 66,67 & 1 & 33,33 \\
Ceftiofur & 10 & 7 & 70 & 3 & 30 & 6 & 5 & 83,33 & 1 & 16,67 \\
\hline
\end{tabular}

*Eficacia clínica y reproductiva: se obtiene mediante método descriptivo en la evaluación de los animales considerados sanos post tratamiento.

Los resultados del tratamiento antibiótico en las yeguas con endometritis se presentan en la Tabla 1. En general, más del $70 \%$ de los animales tratados llegaron a recuperarse de la infección uterina, independientemente del antibiótico 
empleado. Se determinó que los tres protocolos antibióticos propuestos tuvieron una buena eficacia clínica frente a las bacterias aisladas ya que presentaron valores mayores a 70\%, así mismo, se obtuvo una eficacia reproductiva buena ya que más del $60 \%$ de los animales tratados y que no presentaron infección persistente llegaron a tener una preñez.

\section{DISCUSION}

Durante mucho tiempo se ha cuestionado la eficacia de los tratamientos antibióticos dependiendo de la vía de administración (8). Se formulan hipótesis que suponen que las terapias intrauterinas presentan mayor eficacia debido al contacto directo del principio activo con el agente bacteriano (9), así mismo también se considera que las terapias sistémicas evitan efectos secundarios agresivos para el endometrio (10). Se han realizado estudios como los hechos por Cervantes et al (11) en el cual se hizo la administración vía sistémica y no detectaron concentración del antibiótico en el endometrio utilizando $2.0 \mathrm{mg} / \mathrm{kg}$ de ceftiofur durante cinco días consecutivos (11), lo cual contrasta con el estudio de Jonker (12) quien administró una dosis de $2.0 \mathrm{mg} / \mathrm{kg}$, encontrando concentraciones en el endometrio, una hora después de la administración del medicamento (12).

De acuerdo con un estudio publicado por Deborah Nash et al (13), en la actualidad una de las mejores opciones en las terapias antibióticas en infusiones intrauterinas es el ceftiofur, especialmente frente a infecciones causadas por Streptococcus equi zooepidemicus (13). Esta afirmación coincide con un estudio realizado por Collard (14), en el que se demostró que el ceftiofur excede el mínimo de CMI (concentración mínima inhibitoria) para el Streptococcus equi zooepidemicus a partir de dosis de $6.6 \mathrm{mg} / \mathrm{kg}$, generando una alta eficacia clínica contra este microorganismo (14). En la presente investigación se aisló Streptococcus spp. en 5 yeguas, las cuales fueron tratadas con ceftiofur donde se obtuvo una eficacia clínica del 100\%. Scolfield et al (15), menciona que las terapias intrauterinas con ceftiofur generan altos porcentajes de recuperación en yeguas con endometritis bacteriana causada por Streptococcus equi zooepidemicus (15).

Si bien es cierto que la eficiencia reproductiva no depende exclusivamente de los antibióticos, es importante mencionar que una adecuada terapia antimicrobiana permitirá una adecuada resolución del compromiso inflamatorio infeccioso llevando a mayor probabilidad de preñez en las yeguas. Collard et al.(14), describe que una de las principales características del ceftiofur es su acción directa sobre las bacterias productoras de $\beta$-lactamasa, lo cual permite gran eficacia contra $S$. zooepidemicus y E. coli, entre otros agentes microbianos causantes de infecciones en el tracto reproductivo de las yeguas (14). En este estudio, 10 yeguas fueron tratadas con ceftiofur a dosis total de $1.5 \mathrm{~g}$ en infusión intrauterina, obteniéndose una eficacia clínica en 7 casos (70\%). De estas, 6 fueron servidas y 5 quedaron preñadas (83.33\%) considerando un alto valor de preñez para el protocolo con este antibiótico.

La gentamicina ha sido uno de los antibióticos más utilizados en el tratamiento de las infecciones endometriales (16). Ferris RA (6) propone terapias intrauterinas, las cuales inicialmente se basan en una infusión con cloruro de sodio al 0.9\% o lactato de ringer (6), lo cual ayudará a remover la carga bacteriana y los desechos inflamatorios presentes en el útero, ya que estos pueden aumentar la reacción inmunológica a nivel endometrial generando mayor irritación e inflamación (17); posterior a esto se propone el uso de agentes ecbólicos como oxitocina (5-20 UI/IM) o cloprostenol (250 $\mu$ g IM), Ferris afirma que una de las terapias antibióticas que se utilizan en infusiones intrauterinas se basa en la administración de Gentamicina a una dosis total de 1 - 2 gr diluidos en 10 - 20 ml de solución salina (6). Hurtgen (5), afirma que las infusiones intrauterianas con gentamicina pueden generar un mayor índice de inflamación e irritación de la mucosa vaginal (determinado por histopatología) de yeguas sometidas a infusiones intrauterinas cada 24 horas durante 3 días (5).

Durante el actual estudio se realizaron infusiones intrauterinas con gentamicina utilizando una dosis de 1 gr total, en 20 yeguas y les fue instaurado este protocolo antibiótico del cual se encontró que el 75\% (15/20) de las yeguas no presentaban endometritis bacteriana posterior al tratamiento, a su vez, de estas fueron servidas 13 con un porcentaje bueno de preñez 61.54\% (8/13), por lo cual la terapéutica anterior generó índices óptimos en la eficacia clínica y reproductiva de las yeguas.

La penicilina G es un antibiótico ampliamente utilizado en el tratamiento de las infecciones reproductivas en yeguas (18); sin embargo, los datos encontrados en el presente estudio mostraron que 3 de las 4 yeguas tratadas con este antibiótico no presentaron infección postratamiento, y dos de ellas quedaron gestantes. Aunque se evidencia una buena eficacia clínica en las yeguas tratadas, se debe considerar un número mayor de yeguas tratadas con el fin de determinar de manera más certera la eficacia del antibiótico en los tratamientos intrauterinos en yeguas con endometritis.

Rodríguez et al (19) mencionan que la amikacina es uno de los antibióticos con mayor eficacia en la infección uterina debido a su acción bactericida contra organismos gram negativos y su resistencia a enzimas bacterianas (19); así mismo, 
Pedersoli et al (20) afirman que la amikacina alcanza su mayor eficiencia en infusiones intrauterinas en dosis de $2.0 \mathrm{~g}$, con lo cual excede la concentración inhibitoria mínima para bacterias como Pseudomonas y E. coli (20).

Linton and Sertich (21) resalta el amplio espectro de actividad in vitro que tiene la cefquinoma frente a bacterias como Streptococcus spp, Staphylococcus spp, Pseudomonas spp, Escherichia coli, entre otros (21); Los resultados encontrados en el presente estudio sobre el uso clínico reproductivo y la eficacia que presentan las infusiones intrauterinas con cefquinoma representan una nueva posibilidad sobre la terapia clínica de la endometritis bacteriana en yeguas, ya que debido al poco uso farmacológico por esta vía son bajas las tasas de resistencia bacteriana a este agente antibiótico.

En conclusión, la gentamicina fue el antibiótico con mayor eficacia clínica frente a las yeguas diagnosticadas con endometritis, mientras que los animales tratados con ceftiofur mostraron mayor eficacia reproductiva al tener unos mayores índices de preñez.

\section{Declaración de interés}

Los autores declaran que no existe ningún conflicto de interés en este trabajo.

\section{REFERENCIAS}

1. Causey RC. Making Sense of Equine Uterine Infections: The Many Faces of Physical Clearance. Vet J. 2006; 172(3):405421. https://doi.org/10.1016/j.tvjl.2005.08.005

2. Leblanc MM, Causey RC. Clinical and Subclinical Endometritis in the Mare: Both Threats to Fertility. Reprod Domest Anim. 2009; 44(S3):10-22. https://doi.org/10.2746/095777308X357621

3. Riddle WT, LeBlanc MM, Stromberg AJ. Relationships between Uterine Culture, Cytology and Pregnancy Rates in a Thoroughbred Practice. Theriogenology. 2007; 68(3):395-402. https://doi.org/10.1016/j. theriogenology.2007.05.050

4. Overbeck W, Witte TS, Heuwieser W. Comparison of Three Diagnostic Methods to Identify Subclinical Endometritis in Mares. Theriogenology. 2011; 75(7):1311-18. https://doi.org/10.1016/j.theriogenology.2010.12.002

5. Hurtgen JP. Pathogenesis and Treatment of Endometritis in the Mare: A Review. Theriogenology. 2006; 66(3 SPEC. ISS.): 560-566. https://doi.org/10.1016/j.theriogenology.2006.04.006

6. Ferris RA. Therapeutics for Infectious Endometritis: A Clinical Perspective. Rev Bras. Reprod Anim. 2017; 41(1):175179. http://cbra.org.br/portal/downloads/publicacoes/rbra/v41/n1/p175-179\%20(RB634).pdf

7. Buczkowska J, Kozdrowski R, Sikora M, Dzieciol M, Matusz A. Non Traditional Treatments for Endometritis in Mares. Bulgarian Journal of Veterinary Medicine. 2015; 18(4):285-293 https://doi.org/10.15547/bjvm.870

8. Morales PC, Castro RA. Estimación de La Integridad Uterina En Yeguas Pura Raza Chilena y Su Asociación Con Edad y Número de Partos. Revista de Investigaciones Veterinarias del Perú. 2018 29(2): 565. https://doi.org/10.15381/ rivep.v29i2.14489

9. Liu IK, Troedsson MH. The Diagnosis and Treatment of Endometritis in the Mare: Yesterday and Today. Theriogenology. 2008. 70(3):415-20. https://doi.org/10.1016/j.theriogenology.2008.05.040

10. LeBlanc MM. Advances in the Diagnosis and Treatment of Chronic Infectious and Post-Mating-Induced Endometritis in the Mare. Reproduction in Domestic Animals. 2010 45(Suppl. 2):21-27. https://doi.org/10.1111/j.1439$\underline{0531.2010 .01634 . x}$

11. Cervantes CC, Brown MP, Gronwall R, and Merritt K. 1993. Pharmacokinetics and Concentrations of Ceftiofur Sodium in Body Fluids and Endometrium after Repeated Intramuscular Injections in Mares. Am J Vet Res. 1993. 54:573-575. https://pubmed.ncbi.nlm.nih.gov/8484577/ 
12. Jonker FH. Secretion of Ceftiofur in Equine Endometrium after Parenteral Administration. Vet Pharmacol Therap. 1997. 20:(Suppl):37. http://agris.fao.org/agris-search/search.do?recordID=GB1997026008

13. Nash D, Lane E, Herath S, Sheldon M. Endometrial Explant Culture for Characterizing Equine Endometritis. Am J Reprod Immunol. 2008 59(2):105-117. https://doi.org/10.1111/j.1600-0897.2007.00548.x

14. Collard WT, Cox SR., Lesman SP, Grover GS, Boucher JF, Hallberg JW, and Robinson SA and Brown JA. Pharmacokinetics of Ceftiofur Crystalline-Free Acid Sterile Suspension in the Equine. Vet Pharmacol. 2011. 34(5):476-481. https:// doi.org/10.1111/j.1365-2885.2011.01266.x

15. Scofield D, Black J, Wittenburg L, Gustafson D, Ferris R, Hatzel J, Traub - Dargatz J, McCue P. Endometrial Tissue and Blood Plasma Concentration of Ceftiofur and Metabolites Following Intramuscular Administration of Ceftiofur Crystalline Free Acid to Mares. Equine Veterinary Journal. 2014. 46(5):606-610. https://doi.org/10.1111/evj.12192

16. Canisso IF, Coutinho DS. Bacterial Endometritis. Robinson's Current Therapy in Equine Medicine. Saunders, Elsevier; 2015. https://doi.org/10.1016/B978-1-4557-4555-5.00163-1

17. Gores-Lindholm, Alicia R. et al. Relationships between Intrauterine Infusion of N-Acetylcysteine, Equine Endometrial Pathology, Neutrophil Function, Post-Breeding Therapy, and Reproductive Performance. Theriogenology. 2013 80(3):218-227. http://dx.doi.org/10.1016/j.theriogenology.2013.03.026

18. Sosa AM, Mahmoud EA. Ultrasonographic, Cytological and Bacteriological Investigation of Endometritis in Arabian Mares. Global Veterinaria. 15(3):296-303. https://www.idosi.org/gv/gv15(3)15/6.pdf

19. Rodriguez JS, et al. Consequences of Intrauterine Enrofloxacin Infusion on Mare Endometrium. Journal of Equine Veterinary Science. 2012; 32(2):106-111. http://dx.doi.org/10.1016/i.jevs.2011.08.003

20. Pedersoli WM, Fazeli MH, Haddad NS, Ravis WR, Carson RL. Eudometrial AmY Serum Gentamicin Conwn-Tration in Pony .Xares given Repeated Intrauterine Infusion. Am J Vet Res. 1985; 46(5):1025-1028. https://www.ncbi.nlm.nih. gov/pubmed/4003879

21. Linton JK, Sertich PL. The Impact of Low-Volume Uterine Lavage on Endometrial Biopsy Classification. Theriogenology. 2016; 86(4):1004-1007. http://dx.doi.org/10.1016/j.theriogenology.2016.03.028 